\title{
Electrochemical studies of capacitance in cerium oxide thin films and its relationship to anionic and electronic defect densities
}

\author{
William C. Chueh and Sossina M. Haile* \\ Received 3rd June 2009, Accepted 14th July 2009 \\ First published as an Advance Article on the web 27th July 2009 \\ DOI: $10.1039 / \mathbf{b} 910903 j$
}

Small polaron carrier density in epitaxial, doped $\mathrm{CeO}_{2}$ thin films under low oxygen partial pressure was determined from electrochemically-measured capacitance after accounting for interfacial effects and shown to agree well with bulk values.

The electronic properties of thin film oxides are relevant in a broad range of phenomena and devices. ${ }^{1-7}$ In many instances, these properties are strongly influenced by point defect formation and the extent of deviation of the material stoichiometry away from that of the perfect crystal. ${ }^{8-12}$ This strong connection between material chemistry and physical properties has motivated the development of advanced techniques, such as electron energy-loss spectroscopy and positron annihilation spectroscopy, ${ }^{13,14}$ for accurately measuring nonstoichiometry in thin films. Nevertheless, the small sample mass and the constraints of a substrate continue to hinder such measurements. Even a relatively straightforward Hall effect measurement is precluded in the case of low mobility materials. Thus, a reliable and precise method for determining nonstoichiometry and carrier concentration in thin film oxides may be useful for a number of fields.

In this work, we show that it is possible to determine oxygen nonstoichiometry $(\delta)$ in situ, which, in turn, can yield electronic carrier concentration, from an analysis of the capacitance of thin film oxides measured by electrochemical impedance spectroscopy in a cross-plane configuration. The conventional dielectric capacitance is given by

$$
C_{\text {diel }}=\frac{\partial q}{\partial \phi}
$$

where $q$ is the accumulated charge and $\phi$ is the applied electric potential. In the case of a metal oxide that undergoes a change in oxygen content in response to a change in oxygen chemical potential, the electrical equivalent of the chemical driving force, $\mu_{i}^{*}=\frac{\mu_{i}}{e z_{i}}$ (where $e z_{i}$ is the charge on species $i$ and $\mu_{i}$ is the chemical potential of species $i$ ), similarly induces a change in stored charge. ${ }^{15-22}$ For a slab sample that is spatially homogenous, the accumulated charge per species is

$$
q_{i}=e z_{i} c_{i} A L
$$

where $c_{i}$ is the concentration, and $A$ and $L$ are the sample cross-section area and thickness, respectively. In the

Department of Materials Science, California Institute of Technology, Pasadena,CA 91125,USA.E-mail:smhaile@caltech.edu

limits of ideal solution behavior and local electroneutrality $\left(\sum_{i} e z_{i} c_{i}(x)=0\right)$, these definitions imply a chemical capacitance given by

$$
C_{\text {chem }}=\frac{e^{2}}{k_{\mathrm{B}} T} A L\left(\sum_{i} \frac{1}{z_{i}^{2} c_{i}}\right)^{-1}
$$

where $k_{B}, T$ and $e$ have their usual meanings. Thus, a measurement of $C_{\text {chem }}$ can yield the concentration of charged species.

In a sufficiently thick film or bulk sample the area-specific chemical capacitance is several orders of magnitude larger than the interfacial capacitances at gas/solid and solid|solid boundaries. For a typical $0.1 \mathrm{~cm}$ thick nonstoichiometric oxide, the former falls in the range of 1 to $10^{2} \mathrm{~F} \mathrm{~cm}^{-2}$, whereas the latter is typically $10^{-3}$ to $10^{-5} \mathrm{~F} \mathrm{~cm}^{-2} \cdot{ }^{17}$ As a result, the chemical capacitance can be treated as the prevailing capacitance and the interfacial capacitance ignored. In thin films, however, although the relative magnitudes of the capacitance terms are not a priori known, the interfacial capacitance, which is independent of film thickness, can become significant relative to the chemical capacitance, which is proportional to thickness. Difficulties in extracting the individual contributions to the observed capacitance as well as other experimental challenges have precluded establishment of a rigorous link between thin film chemical capacitance and carrier concentration. ${ }^{23-28}$

We show here that by varying the film thickness, or, alternatively, the oxygen partial pressure (more specifically, the oxygen activity), we can deconvolute chemical and interfacial capacitance terms. The study is carried out using epitaxial, nonstoichiometric samarium doped cerium oxide $\left(\mathrm{Sm}_{\mathrm{x}} \mathrm{Ce}_{1-\mathrm{x}} \mathrm{O}_{2-\frac{1}{2} \mathrm{x}-\delta}, \mathrm{SDC}\right)$, a wide band gap material for which the thermodynamics of electron carrier formation are well characterized and the defect chemistry is well known. ${ }^{29-31}$ Under low oxygen partial pressures, both localized electronic defects (small polarons) ${ }^{32,33}$ and oxygen vacancies are relevant for the transport properties of acceptor doped cerium oxide, and the material becomes a mixed ion and electron conductor (MIEC). The reaction for the formation of ionic and electronic charge carriers can be written as:

$$
2 \mathrm{Ce}_{\mathrm{Ce}}^{\mathrm{x}}+\mathrm{O}_{\mathrm{O}}^{\mathrm{x}} \leftrightarrow \frac{1}{2} \mathrm{O}_{2}(\mathrm{~g})+\ddot{\mathrm{V}_{\mathrm{O}}}+2 \mathrm{Ce}_{\mathrm{Ce}}^{\prime}
$$

where the Kröger-Vink notation is used. Under equilibrium conditions and in the dilute limit, the electron concentration, 
$c_{\text {eon }}$, and the oxygen vacancy concentration, $c_{\text {ion }}$, are related to the oxygen partial pressure, $p_{O_{2}}$, according to:

$$
\begin{aligned}
\exp \left(-\frac{\Delta G_{r}}{k_{\mathrm{B}} T}\right) & \approx \frac{1 / 2 c_{\mathrm{eon}}^{2} c_{\mathrm{dop}} p_{O_{2}}^{1 / 2}}{\left(c_{\mathrm{eon}}^{0}-c_{\mathrm{dop}}\right)^{2}\left(c_{\mathrm{ion}}^{0}-1 / 2 c_{\mathrm{dop}}\right)\left(p_{O_{2}}^{0}\right)^{1 / 2}} \\
& =K^{\prime} c_{\mathrm{eon}}^{2} p_{O_{2}}^{1 / 2}
\end{aligned}
$$

where $\Delta G_{r}$ is the standard Gibbs free energy of the reduction of Ce from the $4+$ to the $3+$ valence state, $c_{\text {dop }}$ is the trivalent dopant $(\mathrm{Sm})$ concentration, symbols with superscript " 0 " designate the standard states $\left(p_{O_{2}}^{0}=1 \mathrm{~atm}\right.$, and $c_{\text {eon }}^{0}$ and $c_{\text {ion }}^{0}$ are given by the concentration of $\mathrm{Ce}$ and $\mathrm{O}$ crystallographic sites, respectively), and $K^{\prime}$ is a constant. The simplification given in (4) applies under conditions at which the oxygen vacancy concentration $\left(c_{\text {ion }}=1 / 2\left(c_{\text {dop }}+c_{\text {eon }}\right)\right)$ is much greater than the small polaron concentration $\left(c_{\text {eon }}=2 \delta c_{\text {eon }}^{0}\right)$, at which, furthermore, the chemical capacitance becomes:

$$
C_{\text {chem }}=\frac{e^{2}}{k_{\mathrm{B}} T} A L\left(\frac{1}{c_{\mathrm{eon}}}+\frac{1}{2 c_{\mathrm{dop}}}\right)^{-1}
$$

Measurement of $C_{\text {chem }}$ in nonstoichiometric MIEC oxide films by impedance spectroscopy is facilitated here by deposition onto both sides of a pure oxygen ion conductor (IC). It has been shown elsewhere, ${ }^{15,17}$ from a solution of the fundamental transport equations, that the analytical impedance response of a MIEC can be rigorously mapped to an equivalent circuit in which $C_{\text {chem }}$ explicitly appears. Modification of that result for the present configuration, in which the bulk electronic and ionic resistances of the MIEC thin film are much smaller than the surface reaction resistance, ${ }^{16,24,34}$ the MIEC|IC interface is taken to be reversible to oxygen ions and blocking to electrons, and the porous metal|MIEC interface is taken to be reversible to electrons, yields an equivalent circuit model that is remarkably simple (Fig. 1, inset), particularly given the complexity of the network in the absence of the pure ionic conductor substrate. ${ }^{15,17}$ Specifically, the circuit here consists of a resistor, $R_{\mathrm{ion}}^{\mathrm{IC}}$, representing the oxygen ion transport property of the substrate, in series with a parallel capacitorresistor subcircuit, in which the capacitor represents the sum of chemical, dielectric and interfacial capacitances and the resistor, $R_{\mathrm{ion}}^{\mathrm{ext}}$, represents the inverse of the linearized surface reaction rate for the electrochemical reaction at the porous metal|MIEC interface.

Thin films of SDC, with thickness ranging from 195 to $2241 \mathrm{~nm}$ (much greater than the Debye length $L_{D}=0.18 \mathrm{~nm}$ to justify use of a 1-D impedance model), were deposited onto both sides of $\mathrm{Y}_{0.16} \mathrm{Zr}_{0.84} \mathrm{O}_{1.92}$ (YSZ) (100), (110) and (111) oriented single crystal substrates via pulsed-laser deposition $\left(\mathrm{KrF}, \sim 2 \mathrm{~J} \mathrm{~cm}^{-2}, 20 \mathrm{~Hz}\right)$ at a substrate temperature of $\sim 650{ }^{\circ} \mathrm{C}$ and under $20 \mathrm{~m}$ Torr oxygen pressure. Electron probe microanalysis confirmed that the film $\mathrm{Sm} / \mathrm{Ce}$ ratio was identical, within error, to that of the $\mathrm{Sm}_{0.15} \mathrm{Ce}_{0.85} \mathrm{O}_{1.925}$ polycrystalline target. Out-of-plane X-ray diffraction (XRD) ( $2 \theta$ scan) showed only peaks corresponding to the substrate orientation and a lattice constant $(5.441 \AA)$ that was independent of film thickness, whereas rocking curve measurements revealed a FWHM of $0.4^{\circ}$ (for the (100) peak). The number
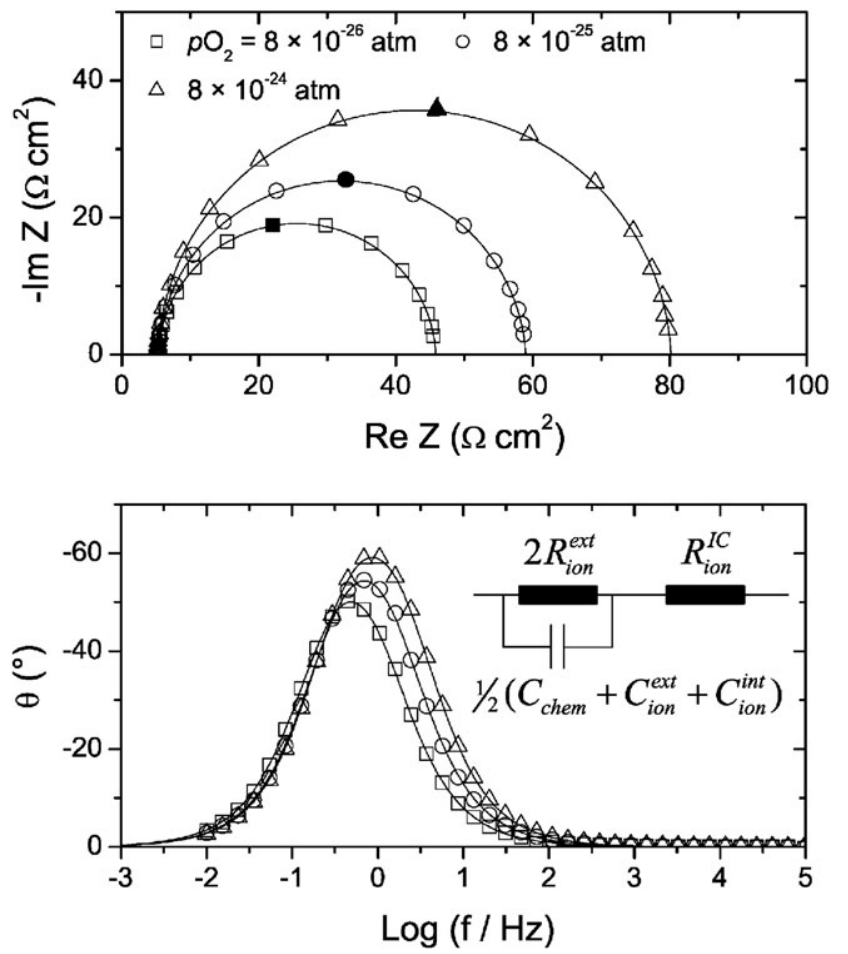

Fig. 1 Typical impedance spectra collected at $650{ }^{\circ} \mathrm{C}$ and various oxygen partial pressures as indicated of $195 \mathrm{~nm}$ samaria doped ceria (SDC) deposited on both sides of a yttria-stabilized zirconia (YSZ) pure ionic conductor. In the Nyquist plot (top), the high frequency intercept corresponds to the resistance of the YSZ substrate and the Pt metal current collectors, the width of the nearly perfect semicircle corresponds to the surface reaction resistance, and the phase shift in the Bode plot (bottom) reflects the combined effect of chemical and interfacial capacitances. The solid symbols in the Nyquist plot shows the impedance at $f=0.19 \mathrm{~Hz}$. The inset shows the physically derived equivalent circuit used to fit the data.

and position of diffraction peaks observed by in-plane XRD ( $\varphi$ scan) was consistent, for each film orientation, with the rotational symmetry of the crystallographic plane normal to the incident beam, indicating alignment registry between the film and the substrate. The epitaxial nature of the SDC thin film was further confirmed by selective area diffraction and dark-field imaging using transmission electron microscopy (TEM); film thickness uniformity was found to be $\pm 4 \%$ by scanning electron microscopy (SEM). The independence of the lattice constant on film thickness suggests that residual stress in these films, despite their epitaxial nature, is small. In the case of molecular beam epitaxy (MBE) grown films, the ceria-YSZ interface has been found to be semi-coherent with the lattice mismatch accommodated mainly by misfit dislocations, ${ }^{35}$ and such a stress-release mechanism may also be operative here; alternatively, stresses in these relatively thick films might not propagate far beyond the interface.

To simulate the electrical measurement conditions, films were annealed at $900{ }^{\circ} \mathrm{C}$ for $24 \mathrm{hr}$. Electron microscopy observations, combined with chemical analysis by energy dispersive spectroscopy, showed insignificant morphological evolution and the absence of detectable cation or dopant interdiffusion between the substrate and the thin film. X-ray 
photoelectron spectroscopy and atomic force microscopy (post heat-treatment) revealed negligible differences in the surface composition and microstructure of the films grown to various thicknesses.

Current collectors were attached by applying Pt paste onto both sides of the SDC $\mid$ YSZ $\mid$ SDC structure and curing for $2 \mathrm{~h}$ at $900{ }^{\circ} \mathrm{C}$. For measurements, the sample was placed in a gas-tight quartz tube positioned inside an electric furnace (500 to $650{ }^{\circ} \mathrm{C}$ ). A mixture of $\mathrm{H}_{2}-\mathrm{H}_{2} \mathrm{O}-\mathrm{Ar}$ or $\mathrm{O}_{2}-\mathrm{Ar}$ (100 $\mathrm{mL} \mathrm{min}^{-1}$ total flow rate, $0.6 \mathrm{~cm} \mathrm{~s}^{-1}$ linear gas velocity at standard conditions) was delivered to the chamber, and the oxygen partial pressure calculated by assuming equilibrium between $\mathrm{H}_{2}, \mathrm{O}_{2}$, and $\mathrm{H}_{2} \mathrm{O}$. The A.C. impedance response was measured at frequencies between 1 or $10 \mathrm{mHz}$ to $10 \mathrm{MHz}$ with a Solartron 1260 A frequency response analyzer at $20 \mathrm{mV}$ amplitude perturbations. Because the cross-plane, rather than the in-plane, electrochemical response is measured in our cell, the current is typically large and not limited by the bulk electrical conductivity of the material (SDC bulk conductivity: $7 \times 10^{-3}$ to $2 \times 10^{-2} \mathrm{~S} \mathrm{~cm}^{-1}$ at $500{ }^{\circ} \mathrm{C}$ for $p_{O_{2}}$ between 0.21 and $2 \times 10^{-31} \mathrm{~atm} .^{17}$

Under all experimental conditions, the impedance response, presented in a Nyquist plot (Fig. 1, top), appeared as a large, nearly perfect, half circle displaced from the origin along the real axis by an amount that corresponds precisely to that expected for the area-normalized resistance of the YSZ single crystal substrate. The impedance response is readily modeled using the physically derived equivalent circuit described above. The constant phase element (CPE) used to represent $C_{\text {tot }}$ showed a relatively large exponent parameter, exceeding 0.93 for all conditions examined, indicating a relatively small dispersion in the relaxation frequency of the system. Physically, the total capacitance, $C_{\text {tot }}$, is the sum of the chemical capacitance $\left(C_{\text {chem }}\right)$, the interfacial (ionic) capacitance at the external SDC/gas electrode interface $\left(C_{\mathrm{ion}}^{\text {ext }}\right)$, and the interfacial (electronic) capacitance at the internal SDC/YSZ interface $\left(C_{\mathrm{eon}}^{\mathrm{int}}\right)$, where a factor of $\frac{1}{2}$ accounts for the presence of the two thin films on both sides of the substrate. The dielectric capacitance $\left(C_{\mathrm{diel}} \sim 10^{-7} \mathrm{~F} \mathrm{~cm}^{-2}\right.$ for a $200 \mathrm{~nm}$ thick SDC film) is more than 4 orders of magnitude smaller than the lowest observed capacitance, and, though in principle a component of $C_{\text {tot }}$, is safely neglected. To satisfy the dilute limit assumption as well as to minimize chemical expansion of SDC upon reduction, $\delta$ was kept below 0.014 during the experiment (corresponding to a chemical expansion $\sim 0.1 \%{ }^{36}$ ). Furthermore, the observed capacitance at a given condition remained unchanged during the course of the experiment after cycling $T$ and $p_{O_{2}}$.

By fitting the parameters of the equivalent circuit to the impedance data, the volume-specific capacitance $\left(C_{\text {tot }} / A L\right)$ was obtained as a function of $p_{O_{2}}$ at temperatures between 500 and $650{ }^{\circ} \mathrm{C}$ for all of the films (Fig. 2). For the thickest film examined (2241 nm, average thickness of films on both sides), the isothermal capacitance is proportional to $p_{O_{2}}^{-1 / 4}$, which is expected if the capacitance is indeed dominated by the chemical capacitance (as implied by eqn (4) \& (5)). However, as the film thickness decreases, the isothermal capacitance curves deviate strongly from the $p_{O_{2}}^{-1 / 4}$ dependence, approaching a constant at higher $p_{O_{2}}$ and lower temperatures. The

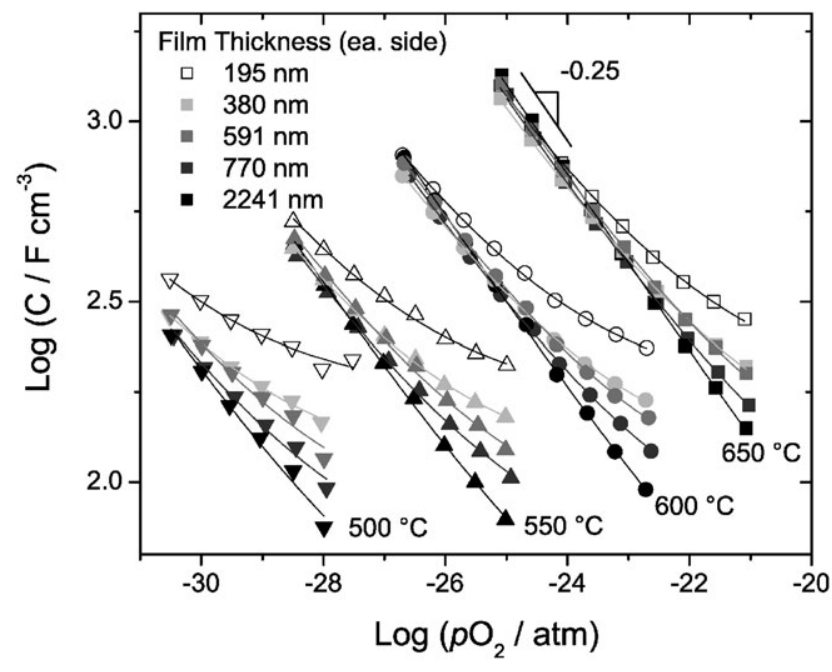

Fig. 2 Dependence of the volume-specific capacitance on oxygen partial pressure for films of varying thickness and at various temperatures, as indicated. Symbols are the measured values and the solid lines are the best fit to eqn (7). As the film thickness decreases, deviation from $p_{\mathrm{O}_{2}}^{-1 / 4}$ dependence increases.

dependence of the volume-specific capacitance on film thickness implies that contributions other than chemical capacitance are indeed present in our system. Given that the volume-specific chemical capacitance depends only on the electron carrier concentration (or equivalently, oxygen nonstoichiometry), the total capacitance can be expressed as:

$$
C_{\mathrm{tot}}=\frac{e^{2} A}{k_{\mathrm{B}} T}\left(\frac{1}{c_{\mathrm{eon}}\left(p_{O_{2}}, T\right)}+\frac{1}{2 c_{\mathrm{dop}}}\right)^{-1} L+C_{\mathrm{int}}\left(p_{O_{2}}, T\right)
$$

where $C_{\mathrm{int}}=C_{\mathrm{ion}}^{\mathrm{ext}}+C_{\mathrm{eon}}^{\mathrm{int}}$ is the sum of interfacial capacitances and it is implicitly assumed that the Gibbs free energy of carrier formation is independent of film thickness, consistent with the low residual stress of these relatively thick films. Fig. 3 shows the capacitance plotted as a function of film thickness and fit to eqn (6). Varying the film thickness while

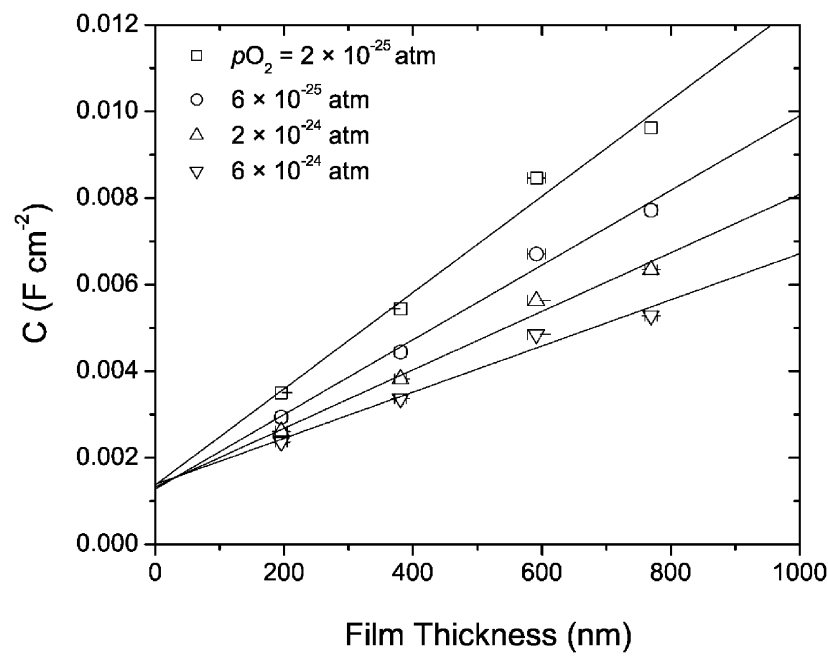

Fig. 3 Capacitance measured at $600{ }^{\circ} \mathrm{C}$ (various oxygen partial pressures) as a function of SDC film thickness. The solid lines are the best fit to eqn (6). The slope gives $C_{\text {chem }} / L$, whereas the intercept gives the sum of interfacial capacitances. 


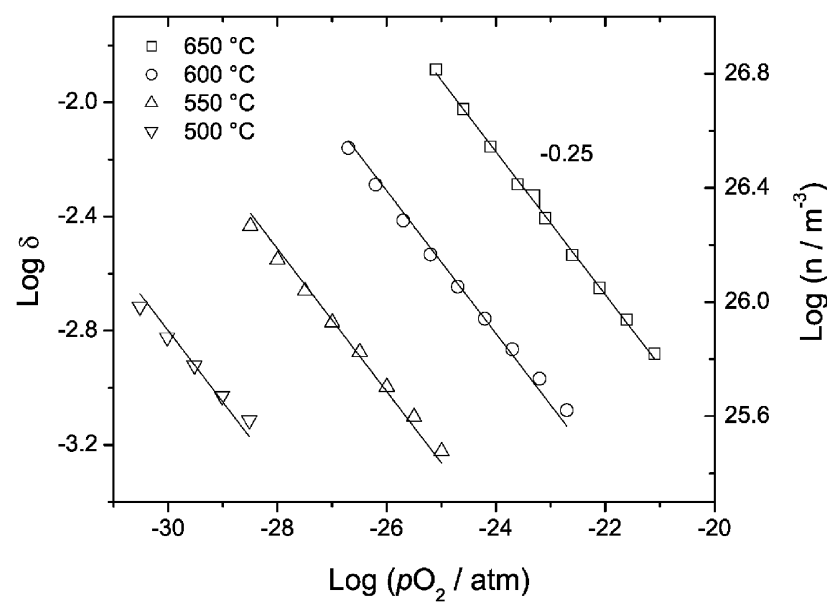

Fig. 4 Oxygen nonstoichiometry $(\delta)$ and electron carrier concentration $\left(c_{\text {eon }}\right.$ or $\left.n\right)$ determined using the film thickness-dependent capacitance data (partially shown in Fig. 3). Solid lines show the fit to $c_{\text {eon }}=2 \delta c_{\text {eon }}^{0}=K^{\prime \prime}(T) p_{O_{2}}^{-1 / 4}$, where $K^{\prime \prime}$ directly yields the standard Gibb's free energy of cerium reduction.

holding the cell area constant permits the extraction of the thickness-dependent chemical capacitance and the thickness-independent interfacial capacitances. The electron carrier concentration and the oxygen nonstoichiometry computed from the chemical capacitance values exhibit excellent agreement with the expected $p_{O_{2}}^{-1 / 4}$ dependence (Fig. 4). Furthermore, the interfacial capacitances obtained from this analysis (not shown) display a very weak $p_{O_{2}}$ dependence and nearly no temperature dependence.

Having demonstrated the $p_{\mathrm{O}_{2}}^{-1 / 4}$ dependence of $C_{\text {chem }}$ and the relatively $p_{\mathrm{O}_{2}}$-independent $C_{\mathrm{int}}$, each isothermal capacitance data set in Fig. 1 can be described according to the following approximation:

$$
C_{\mathrm{tot}}=\frac{e^{2} A L}{k_{\mathrm{B}} T}\left(\frac{1}{c_{\mathrm{eon}}^{*}(T) p_{O_{2}}^{-1 / 4}}+\frac{1}{2 c_{\mathrm{dop}}}\right)^{-1}+C_{\mathrm{int}}(T)
$$

where $c_{\text {eon }}^{*}$ is the $p_{\mathrm{O}_{2}}$-independent component in the polaron concentration $\left(c_{\mathrm{eon}}=c_{\mathrm{eon}}^{*} p_{\mathrm{O}_{2}}^{-1 / 4}\right)$. In contrast to the analysis associated with eqn (6) for which multiple films of differing thicknesses are used to deconvolute capacitance terms, application of eqn (7) requires only the capacitance of one sample measured under several oxygen partial pressures. Utilizing eqn (4) and $c_{\text {eon }}^{*}$ obtained from eqn (7) at multiple temperatures, we compute the $\mathrm{Ce}^{4+} \rightarrow \mathrm{Ce}^{3+}$ standard reduction enthalpy and entropy of SDC reduction according to eqn (3) for each of the films. The thermodynamic quantities (Table 1) are not only essentially independent of film thickness and orientation, but also identical, within experimental error, to the values measured for bulk materials. The agreement demonstrates that the chemical capacitance has been correctly extracted from the total capacitance, and that impedance spectroscopy, under ideal conditions, can be utilized to determine carrier concentrations and thermodynamic quantities even in thin films. The results further imply that our films are essentially bulk-like in nature, with low strain energy, consistent with the thickness independent lattice constant, and minimal influence of low-angle tilt boundaries present in the growth direction, consistent with the narrow FWHM measured in the rocking curve diffraction experiment. In contrast, in very thin films, $10 \mathrm{~nm}$ or less, ${ }^{37}$ and in nanostructured bulk materials ${ }^{38}$ large decreases in reduction enthalpy have been reported, indicating that size and strain effects can, under different circumstances, induce substantial deviations in chemical bonding state.

In summary, we have determined electron carrier concentration and $\delta$ as low as $10^{-3}$ in epitaxial, doped ceria thin films using electrochemical impedance spectroscopy. Interfacial capacitance exceeded $65 \%$ of the observed capacitance for a moderate $\delta$ of $10^{-3}$ in $195 \mathrm{~nm}$ thick SDC, underscoring the importance of extracting bulk and interfacial contributions to capacitance to permit quantification of carrier concentrations. The reduction thermodynamics of our SDC films are in excellent agreement with the behavior of bulk samples. Barring an unlikely situation in which multiple errors conveniently cancel one another, this agreement validates the approach and ultimately points to a systematic methodology for probing the thermodynamics of thin film materials.

\section{Acknowledgements}

The authors gratefully acknowledge the National Science Foundation (DMR-0604004) for funding and Dr Youli Li at the University of California, Santa Barbara for assistance with thin film X-ray diffraction experiments.

Table 1 Comparison of standard enthalpies $\left(\Delta H_{r}\right)$ and entropies $\left(\Delta S_{r}\right)$ for the reduction reaction expressed in eqn (3), on a per mole atomic oxygen basis, obtained in thin film and bulk materials. Electron carrier formation enthalpy is given by $\Delta H_{r} / 2$. Number after "SDC" indicates Sm doping level (\%). Note: ref. 17 employs a slightly different definition of the equilibrium constant of reduction and the thermodynamic quantities have been recalculated in a manner that is consistent with the present work and with ref. 31

\begin{tabular}{|c|c|c|c|c|}
\hline & $\Delta H_{r}(\mathrm{eV})$ & $\Delta S_{r}\left(10^{-3} \mathrm{eV} \mathrm{K}^{-1}\right)$ & Measurement method & Ref \\
\hline SDC15(100) $195 \mathrm{~nm}$ & $4.16 \pm 0.02$ & $1.10 \pm 0.03$ & Thin film impedance & This work \\
\hline SDC15(100) $380 \mathrm{~nm}$ & $4.17 \pm 0.03$ & $1.12 \pm 0.03$ & Thin film impedance & This work \\
\hline $\operatorname{SDC} 15(100) 591 \mathrm{~nm}$ & $4.18 \pm 0.04$ & $1.15 \pm 0.04$ & Thin film impedance & This work \\
\hline SDC15(100) $770 \mathrm{~nm}$ & $4.18 \pm 0.04$ & $1.14 \pm 0.04$ & Thin film impedance & This work \\
\hline SDC15(100) $2241 \mathrm{~nm}$ & $4.18 \pm 0.02$ & $1.17 \pm 0.03$ & Thin film impedance & This work \\
\hline $\operatorname{SDC} 15(110) 2124 \mathrm{~nm}$ & $4.19 \pm 0.06$ & $1.17 \pm 0.07$ & Thin film impedance & This work \\
\hline SDC15(111) $2124 \mathrm{~nm}$ & $4.19 \pm 0.05$ & $1.19 \pm 0.04$ & Thin film impedance & This work \\
\hline SDC15 (polycrystalline) & $4.18 \pm 0.05$ & $1.15 \pm 0.05$ & Bulk impedance & 17 \\
\hline SDC10 (polycrystalline) & 4.15 & 1.10 & Thermogravimetry & 31 \\
\hline SDC20 (polycrystalline) & 3.99 & 1.13 & Thermogravimetry & 31 \\
\hline
\end{tabular}




\section{References}

1 C. H. Ahn, J.-M. Triscone, N. Archibald, M. Decorux, R. H. Hammond, T. H. Geballe, O. Fischer and M. R. Beasley, Science, 1995, 269, 373-376.

2 J. B. Bates, N. J. Dudney, B. Neudecker, A. Ueda and C. D. Evans, Solid State Ionics, 2000, 135, 33-45.

3 A. Gupta and J. Z. Sun, J. Magn. Magn. Mater., 1999, 200, $24-43$.

4 Y. Matsumoto, M. Murakami, T. Shono, T. Hasegawa, T. Fukumura, m. Kawasaki, P. Almet, T. chikyow, S. Koshihara and h. Koinuma, Science, 2001, 291, 854-856.

5 S. J. Pearton, C. R. Abernathy, M. E. Overberg, G. T. Thaler, D. P. Norton, N. Theodoropoulou, A. F. Hebard, Y. D. Park, F. Ren, J. Kim and L. A. Boatner, J. Appl. Phys., 2003, 93, 1-13.

6 K. A. Striebel, C. Z. Deng, S. J. Wen and E. J. Cairns, J. Electrochem. Soc., 1996, 143, 1821-1827.

7 G. D. Wilk, R. M. Wallace and J. M. Anthony, J. Appl. Phys., 2001, 89, 5243-5275.

8 R. J. Cava, H. Takagi, J. J. Krajewski, J. W. F. Peck and H. Y. Hwang, Phys. Rev. B: Condens. Matter Mater. Phys., 1993, 47, 11525-11528.

9 T. Ohnishi, K. Shibuya, T. Yamamoto and M. Kippmaa, J. Appl. Phys., 2008, 103, 103703-103701.

10 M. Suzuki, Jpn. J. Appl. Phys., 1992, 31, 3830-3833.

11 O. N. Tufte and P. W. Chapman, Phys. Rev., 1967, 155, 796-802.

12 S. H. Lee, C. E. Tracy and J. R. Pitts, Electrochem. Solid-State Lett., 2004, 7, 299-301.

13 D. W. Gidley, H.-G. Peng and R. S. Vallery, Annu. Rev. Mater. Res., 2006, 36, 49-79.

14 M. J. Puska and R. M. Nieminen, Rev. Mod. Phys., 1994, 66, 841-893.

15 J. Jamnik and J. Maier, J. Electrochem. Soc., 1999, 146, 4183-4188.

16 J. Jamnik and J. Maier, Phys. Chem. Chem. Phys., 2001, 3, $1668-1678$.

17 W. Lai and S. M. Haile, J. Am. Ceram. Soc., 2005, 88, 2979-2997.

18 J. Maier, Solid State Phenom., 1994, 39-40, 35-60.

19 S. Adler, Chem. Rev., 2004, 104, 4791-4844.

20 I. D. Raistrick, J. R. Macdonald and D. R. Franceschetti, Impedance Spectroscopy, John Wiley \& Sons, Inc., New York, 1987.
21 J. Bisquert, Phys. Chem. Chem. Phys., 2003, 5, 5360-5364.

22 J. Bisquert, F. Fabregat-Santiago, I. Mora-Sero, G. GarciaBelmonte, E. Barea and E. Palomares, Inorg. Chim. Acta, 2008, 361, 684 .

23 J. Fleig, J.-R. Kim, J. Jamnik and J. Maier, Fuel Cells, 2008, 5, 330-337.

24 T. Kawada, J. Suzuki, M. Sase, A. Kaimai, K. Yashiro, Y. Nigara, J. Mizusaki, K. Kawamura and H. Yugami, J. Electrochem. Soc., 2002, 149, E252-E259.

25 G. T. Kim, S. Wang, A. J. Jacobson, Z. Yuan and C. Chen, J. Mater. Chem., 2007, 17, 1316-1320.

26 M. Sase, J. Suzuki, K. Yashiro, T. Otake, A. Kaimai, T. Kawada, J. Mizusaki and H. Yugami, Solid State Ionics, 2006, 177, 1961-1964.

27 G. la O and Y. Shao-Horn, J. Electrochem. Soc., 2009, 156, B816-B824.

28 W. Jung and H. L. Tuller, J. Electrochem. Soc., 2008, 155, B1194-B1201.

29 R. J. Panlener, R. N. Blumenthal and J. E. Garnier, J. Phys. Chem. Solids, 1975, 36, 1213-1222.

30 S. Wang, H. Inaba and H. Tagawa, J. Electrochem. Soc., 1997, 144, 4076-4080.

31 T. Kobayashi, S. Wang, M. Dokiya, H. Tagawa and T. Hashimoto, Solid State Ionics, 1999, 126, 349-357.

32 E. Ruiz-Trejo and J. Maier, J. Electrochem. Soc., 2007, 154, B583-B587.

33 H. L. Tuller and A. S. Nowick, J. Phys. Chem. Solids, 1977, 38, 859-867.

34 F. S. Baumann, J. Fleig, H.-U. Habermeier and J. Maier, Solid State Ionics, 2006, 177, 1071-1081.

35 C. M. Wang, S. Thevuthasan and C. H. F. Peden, J. Am. Ceram. Soc., 2003, 86, 363-365.

36 S. R. Bishop, K. L. Duncan and E. D. Wachsman, Electrochim. Acta, 2009, 54, 1436-1443.

37 O. Costa-Nunes, R. M. Ferrizz, R. J. Gorte and J. M. Vohs, Surf. Sci., 2005, 592, 8-17.

38 Y.-M. Chiang, E. B. Lavik, I. Kosacki, H. L. Tuller and J. Y. Ying, J. Electroceram., 1997, 1, 7-14. 